\title{
Sensorless Sensing in Wireless Networks: Implementation and Measurements
}

\author{
Kristen Woyach, Daniele Puccinelli and Martin Haenggi \\ Network Communication and Information Processing Laboratory \\ Department of Electrical Engineering \\ University of Notre Dame \\ $\{$ kwoyach, dpuccine, mhaenggi\}end.edu
}

\begin{abstract}
Multipath fading and shadowing are usually regarded as negative phenomena hindering proper radio communication. Adopting a completely different stance, this paper illustrates that such phenomena enable information harvesting from received signal strength leading to a number of original applications requiring no conventional sensing hardware. The radio itself, provided that it can measure the strength of the incoming signal, is the only sensor we use; with this sensorless sensing approach, any wireless network becomes a sensor network. We show that motion of the nodes in the network or motion of bodies external to the network leaves a characteristic footprint on signal strength patterns, which may be exploited for motion detection. We illustrate a technique to extract an estimate of velocity from signal strength, and we leverage on the spatial memory properties of wireless links to present a method for spatial configuration recognition.
\end{abstract}

\section{INTRODUCTION}

Extracting information from the variations in the strength of received signals is a simple but powerful way to turn any wireless network into a sensor network. Physical phenomena such as small-scale fading and shadowing affect signal strength; as long as everything is static, received signal levels are expected to be constant, but they fluctuate when motion is introduced. This is particularly evident in rich scattering environments, such as offices or hallways; in this paper, we will restrict our attention to such indoor locales. We establish the basis for sensorless motion detection through the exploitation of multipath fading and shadowing effects in Section II. We build on this concept in Section III, where we present applications to velocity detection. In Section IV, we utilize the spatial nature of multipath fading to underscore the memory properties inherent to wireless links. Finally, as a side note, we provide some insight into the conditions which prevent such links from being symmetric.

We will illustrate these points with a number of examples using two different hardware platforms: Crossbow's MICA2 and MICAz. The MICA2 platform is equipped with an Atmel ATmega128L and has a narrowband radio (precisely, a $433 \mathrm{MHz}$ Chipcon CC1000 transceiver). Since the MCU is to handle medium access and baseband processing, a finegrained event-driven real-time operating system (TinyOS [1]) has been implemented to specifically address the concurrency and resource management needs of sensor nodes. The MI$\mathrm{CAz}$ platform has the same processor as MICA2 and comes

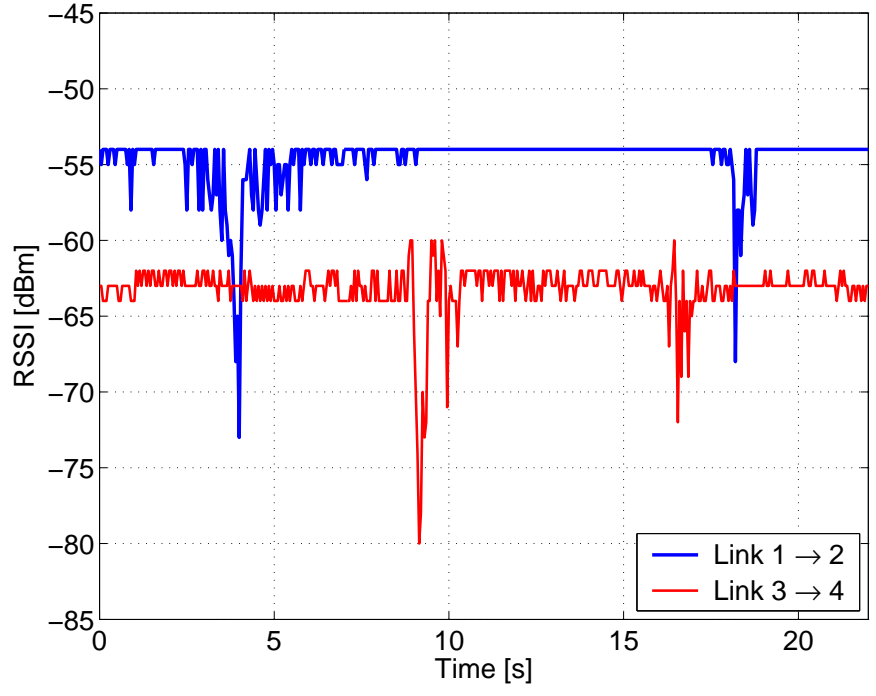

Fig. 1. Motion detection leading to velocity estimation: an example with MICAz nodes arranged as depicted in Fig. 2.

equipped with an IEEE 802.15.4-compliant 2.4GHz Chipcon CC2420 radio which uses spread spectrum techniques to increase channel reliability and noise tolerance by spreading the signal over a wide range of frequencies. In all the experiments featured in the paper, we use a transmit power of $10 \mathrm{dBm}$ with MICA2 and 0dBm with MICAz unless otherwise stated.

\section{MOTION DETECTION}

Motion of wireless nodes with respect to each other causes continuous topology changes in the network. Given a link between a transmitter and a receiver, the level of the received signal strength (whose indication is typically referred to as RSSI) varies considerably as a function of the position of the terminals in the environment. This is mainly due to multipath fading effects as well as interruptions of the lineof-sight path (shadowing). We informally refer to the RSSI level in a given location as the quality of the fading spot occupied by a terminal. If a terminal is continuously moving, it occupies a series of fading spots with different levels of quality. Therefore, as long as a terminal can detect such variations, it can detect its own motion or the motion of objects 


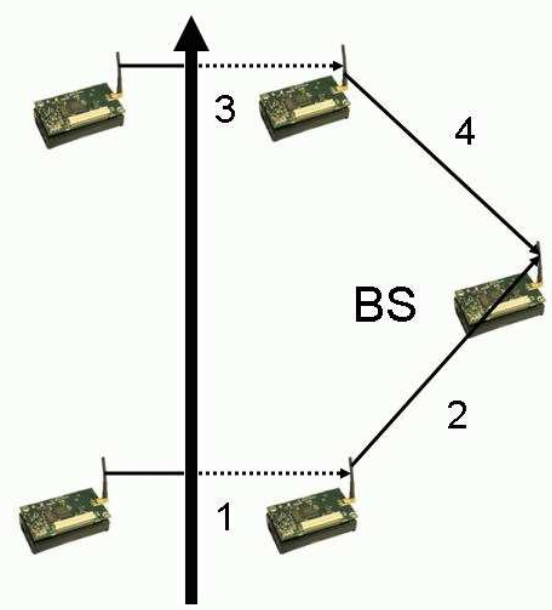

Fig. 2. Motion between the lower node pair disrupts the line-ofsight path causing shadowing (1); motion is detected on the basis of the RSSI and the base station is notifi ed (2). Motion between the upper pair is likewise detected (3) and relayed (4). Given the distance between node pairs, the base station can estimate the velocity of the moving body.

in its surroundings.

To better illustrate this concept, we place two receiver/transmitter pairs of $\mathrm{MICAz}$ motes $6 \mathrm{~m}$ apart at an elevation of $50 \mathrm{~cm}$ above the floor, as shown in Fig. 2. For each pair, the transmitter and the receiver are $1.5 \mathrm{~m}$ apart. The receivers in each pair measure the signal strength and forward it to a base station. On the sole basis of RSSI, it is possible to determine when the line-of-sight path is broken; in this sense we speak of sensorless motion detection. Fig. 1 shows the variations in the measured signal strength for both links as the same person moves between the members of each pair. By comparing the time between subsequent shadowing events, it is possible to estimate the speed of the person as she first walks down the hallway and then runs back to her starting position.

In a rich scattering environment (such as the one depicted in Fig. 3(a)), both the motion of a node and the motion in the surroundings cause variations in signal strength. The motion of a node causes small-scale fading which affects all multipath components and produces continuous variations in the network topology causing noticeable changes in a number of wireless links. Fig. 3(b) shows an example of the impact of the motion of a transmitter in a point-to-point scenario. Motion in the environment, on the other hand, affects the signal strength measured by the nodes in a twofold fashion. Motion in the vicinity of nodes causes small-scale fading, resulting in variations of up to $\pm 10 \mathrm{~dB}$; however, larger variations can be caused by objects moving through line-of-sight paths, which cause shadowing effects. Since motion in the environment only affects a limited number of paths, it is expected to produce smaller variations in the RSSI. Fig. 3(c) shows an example of the effect of the motion of a piece of furniture in an indoor

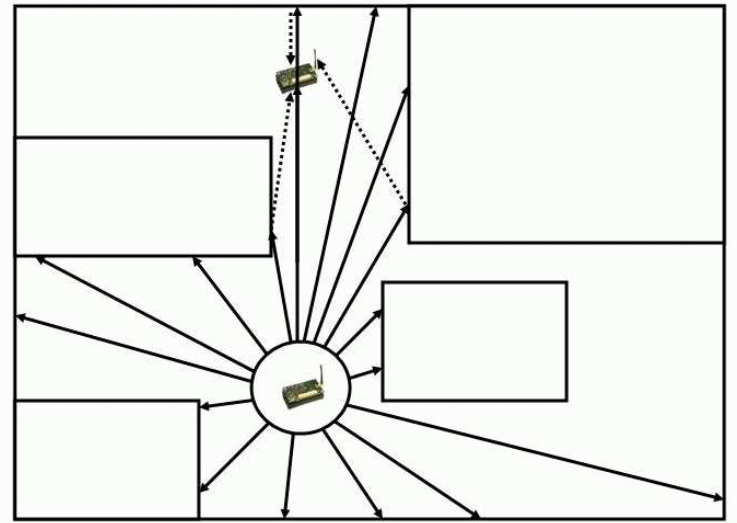

(a) Two sensing nodes in an indoor environment with a line-of-sight as well as reffected paths.

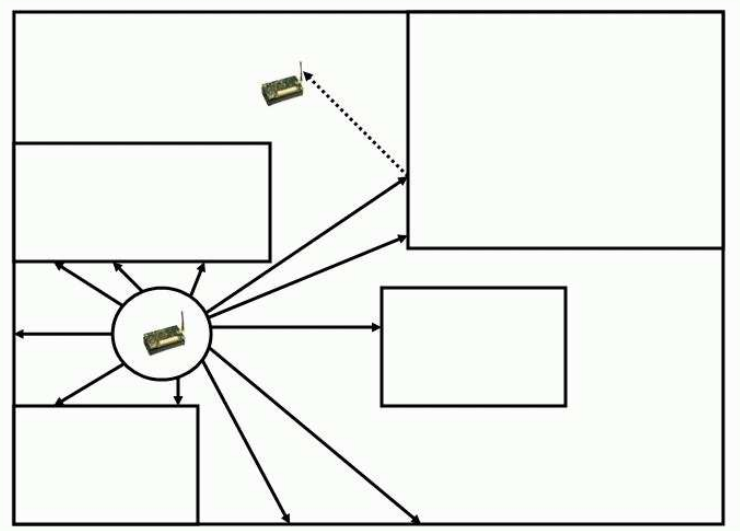

(b) After the transmitter has moved, there remain only refected paths.

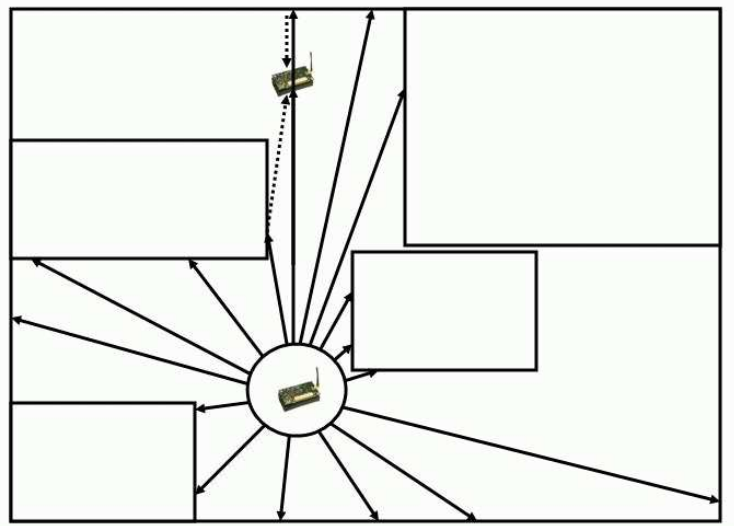

(c) After a modifi cation in the topology of the surroundings, fewer paths can reach the receiver.

Fig. 3. The motion of a node or a change in the surroundings of the nodes can affect communication between sensing nodes. The arrows show radio wave propagation through the wireless channel; solid lines indicate sample direct paths, and dashed lines indicate sample refected paths. 

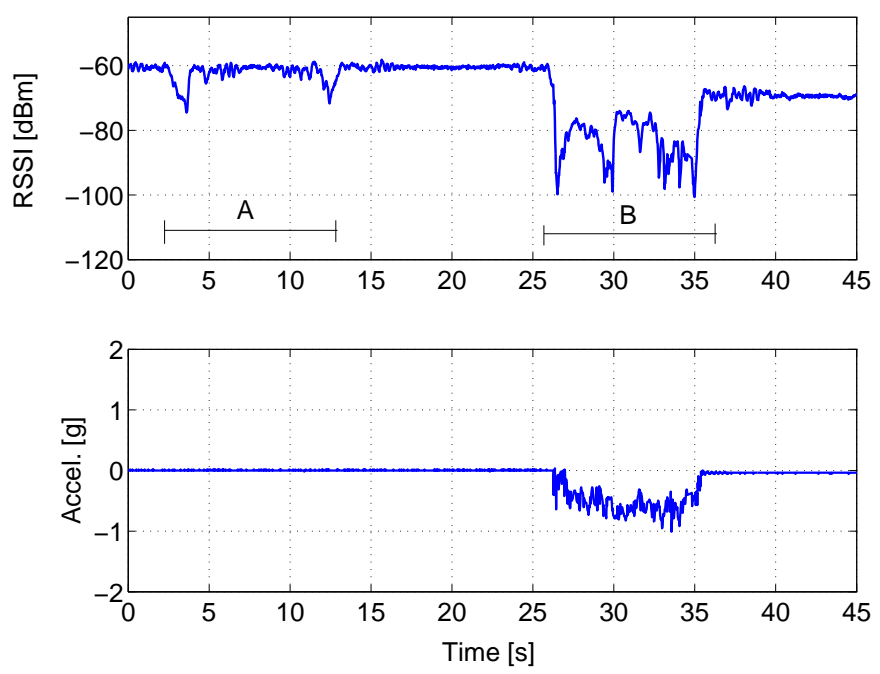

Fig. 4. Motion in the environment (A) versus motion of MICA2 terminals (B).
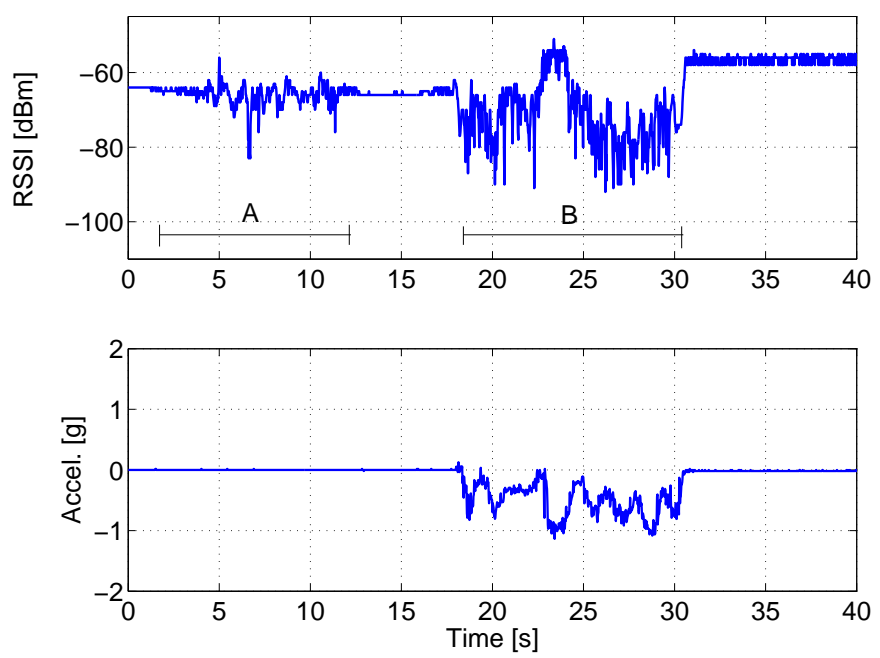

Fig. 5. Motion in the environment (A) versus motion of $\mathrm{MICAz}$ terminals (B).

locale, which in this case blocks one of the reflected paths, thus affecting the signal strength at the receiver.

The variation in RSSI caused by the motion of network nodes and by the motion in the environment are compared in Figures 4 and 5, where RSSI and accelerometer readings are shown for acquisitions with the MICA2 and MICAz platforms. In these experiments, a receiver/transmitter pair of motes is placed in a hallway. The strength of the signal measured by one node as different motion events occur is shown along with the readings from an accelerometer connected to the transmitter. At first, one person walks down the hallway along the line-ofsight path; in this case, although the signal strength undergoes considerable variations, there are no changes in the signals from the accelerometer. The received signal strength becomes constant as the person stops moving, but the changes resume
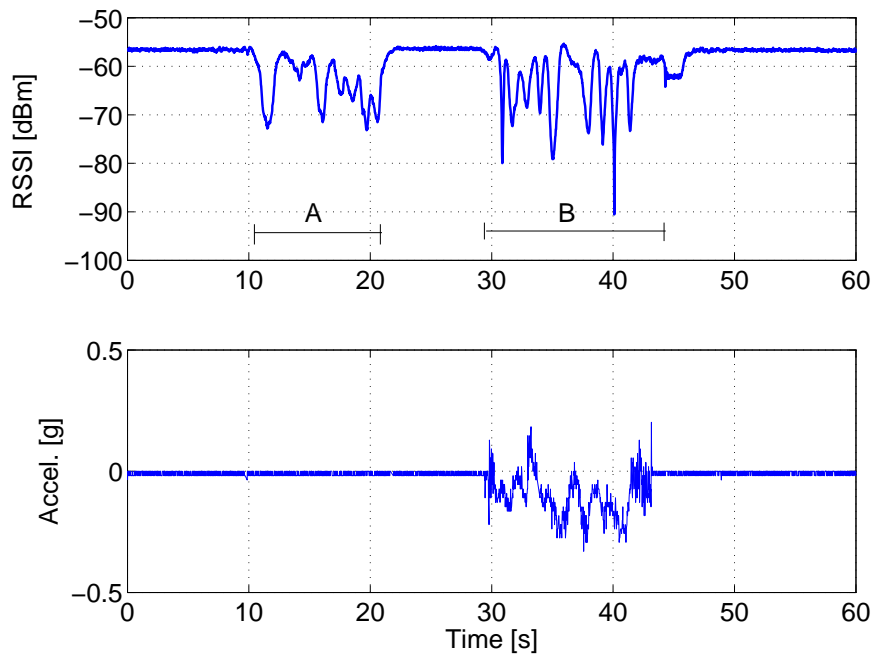

Fig. 6. Motion in the surroundings of MICA2 nodes (A) yields similar effects as the motion of the terminals themselves (B) due to the particular layout of the environment where the nodes are deployed. However, the motion of the terminals still produces larger fluctuations.
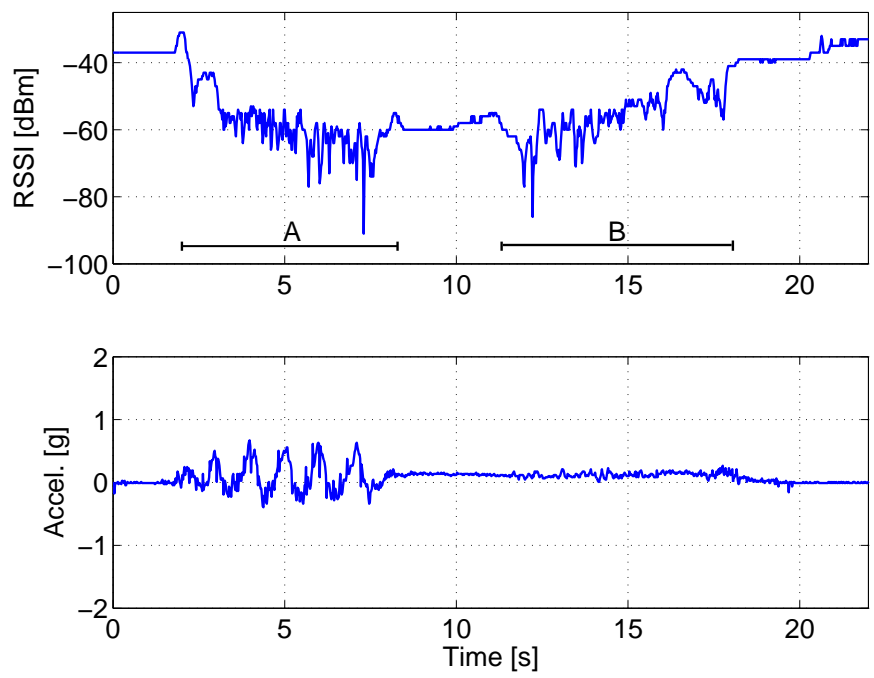

Fig. 7. A MICAz transmitter as a more robust motion sensor than an accelerometer. The erratic motion (A) is observable by the accelerometer, but if the changes are below the sensitivity of the accelerometer (B), motion is observable only by fluctuations in the RSSI.

when the transmitter starts moving around the hallway. The RSSI variations are more consistent and more pronounced in the case of node motion. This is visually apparent in the examples and is quantifiable by determining the standard deviation of the $\mathrm{dB}$ values of the RSSI. We use this nonstandard method to assign a stronger weight to small power values which would not influence the value of the standard deviation. For Fig. 5, the case of motion in the environment exhibits a standard deviation of $3.3 \mathrm{~dB}$ while motion of the node produces a standard deviation of $8.27 \mathrm{~dB}$. It is worth pointing out that the nodes do not terminate their motion in 

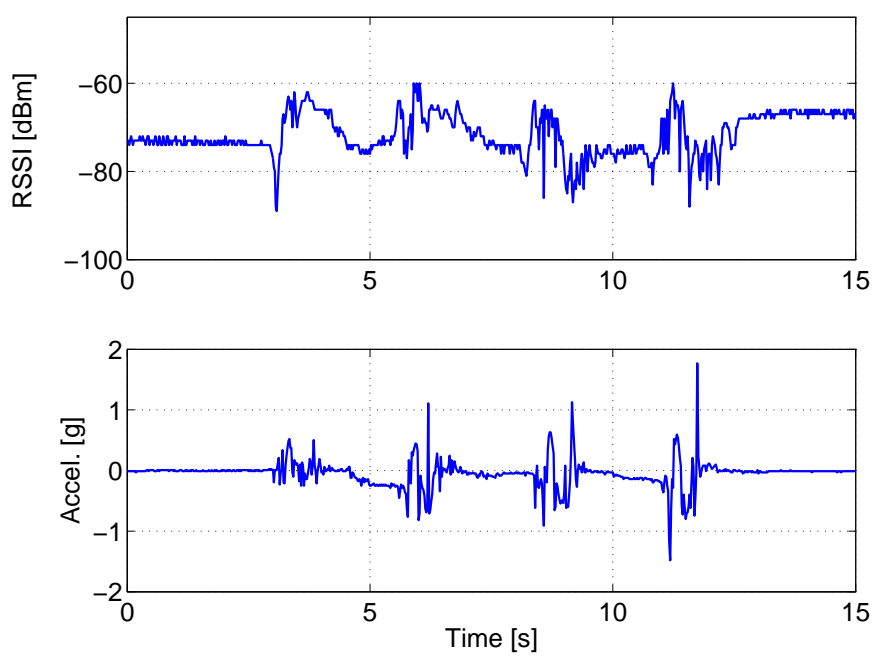

Fig. 8. A MICAz transmitter used as a pedometer: in this case, the RSSI pattern indicates that four steps were taken, as confi rmed by the accelerometer readings.

their initial position, which accounts for the difference in the RSSI levels before and after the second motion event in both Fig. 4 and Fig. 5.

The magnitude of the fluctuations due to motion in the surroundings of the network strongly depends on the trajectory of such motion with respect to the network topology and the layout of the environment where the nodes are deployed. For instance, in Fig. 6, the causes of RSSI variation are visually harder to distinguish without the accelerometer readings. However, the $\mathrm{dB}$ standard deviation in the case of node motion is still larger $(5.6 \mathrm{~dB}$ for the case of node motion and $4.8 \mathrm{~dB}$ for the case of motion in the environment).

Fig. 7 presents a comparison of the sensitivity of an accelerometer-based versus an RSSI-based motion detector. In this experiment, a MICAz transmitter is carried across a room in an erratic fashion, and then is carried back with nearly constant velocity. The first motion event is clearly observable with both RSSI measurements and accelerometer readings. However, when the changes in velocity are very small, the accelerometer cannot easily track this movement while the RSSI still exhibits large fluctuations. Although this RSSI-based motion detection may not replace an actual accelerometer, it certainly contributes valuable information in many cases.

We now illustrate an interesting example of the kind of information that can be harvested from signal strength. A MICAz transmitter equipped with an accelerometer attached to a person's foot continuously sends packets to a static receiver that measures signal strength. In Fig. 8, the received signal strength is compared to the readings from the accelerometer. Aperiodic fluctuations in received signal strength provide a qualitative indication of acceleration which in this case allows us to conclude that the person made exactly four steps.
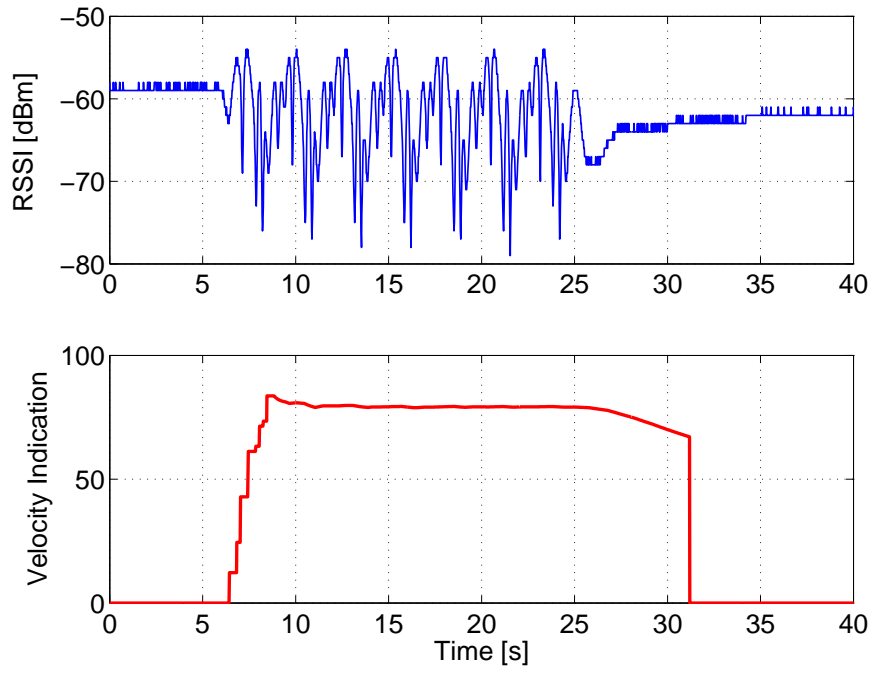

Fig. 9. Estimating the velocity of a moving node on a motorized turntable.

\section{VELOCITY ESTIMATION}

The goal of this application is to give a rough assessment of the velocity of a moving terminal using a point-to-point wireless connection. A continuous exchange of packets between a moving node and a static base station allows the latter to harvest information regarding the velocity of the former based on the pattern of the received signal strength. A displacement of $\lambda / 2$ of the moving node causes a noticeable change in the RSSI pattern; therefore, velocity information can be obtained by tracking the frequency of such changes with a simple signal processing algorithm.

As an example, we use an algorithm based on the analysis of the extrema of the RSSI. After processing the raw signal with a moving average filter to remove noise, the extrema are identified by sign reversals in the first-order difference. The frequency of change of the fading pattern can be determined by computing the time difference between successive extrema; for a constantly moving transmitter, this time difference would be sufficient for velocity estimation. However, when the transmitter is not moving, there are no extrema to detect; in order to account for this case, we define a maximum wait time between extrema. The velocity indication output by the motes is the ratio in percent of the current time difference to the maximum time difference between extrema.

To illustrate this approach, we use MICA2 motes operating at a carrier frequency of $433 \mathrm{MHz}$, which corresponds to a wavelength of $69 \mathrm{~cm}$; the transmitter is programmed to send out a packet every $20 \mathrm{~ms}$. With these parameters, small velocities $(<2.5 \mathrm{~m} / \mathrm{s})$ can be accurately estimated. To correlate the velocity indication with linear velocity levels, we place a transmitter on a motorized turntable with a radius of $66 \mathrm{~cm}$, and a receiver at approximately the same height located in the same room. This setup is shown in Fig. 10. By changing the supply voltage of the DC motor controlling the turntable, we 


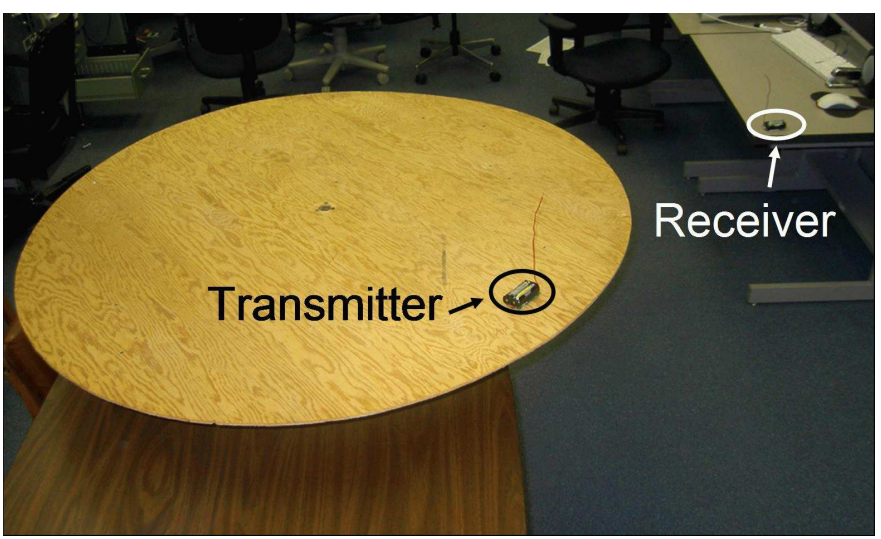

Fig. 10. Turntable setup for velocity estimation.

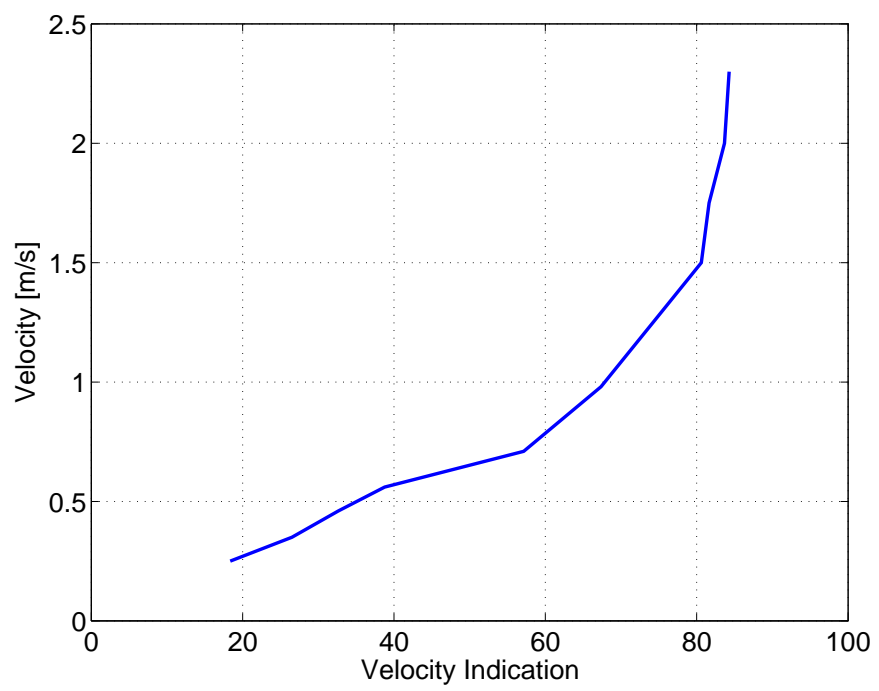

Fig. 11. Velocity as a function of the indication given by the motes.

are able to obtain different, known velocity levels. The use of the turntable also produces a periodic fading pattern, thus ensuring repeatability. Fig. 9 displays the strength of the signal received by the fixed terminal along with the corresponding velocity indication for one of the configuration tests. Fig. 11 shows the mapping of this indication to linear velocity levels. In the case of Fig. 9, velocity is estimated at $1.5 \mathrm{~m} / \mathrm{s}$.

We then estimate the velocity of a general case by attaching the MICA2 mote to a toy car. The result of this test is shown in Fig. 12. After moving down a hallway at a high speed, estimated by the receiver to be $2.3 \mathrm{~m} / \mathrm{s}$, the car comes back at a slower speed, estimated to be $0.27 \mathrm{~m} / \mathrm{s}$.

\section{SPATIAL MEMORY}

\section{A. Spatial nature of fading}

Multipath fading and shadowing make wireless links very sensitive to changes in the environment and form the underlying cause of time-varying packet delivery performance
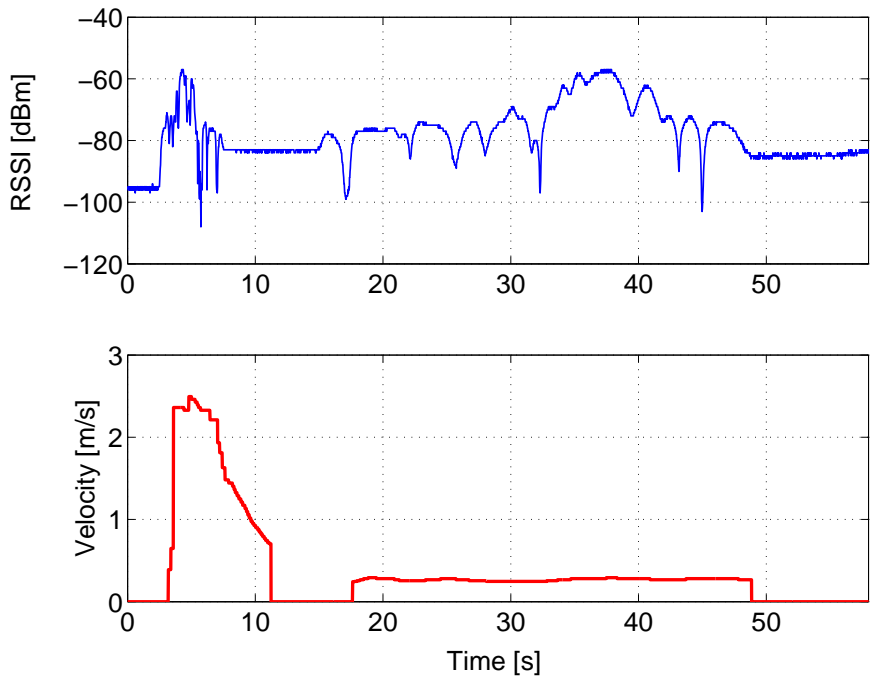

Fig. 12. Estimating the velocity of a moving node in a hallway.

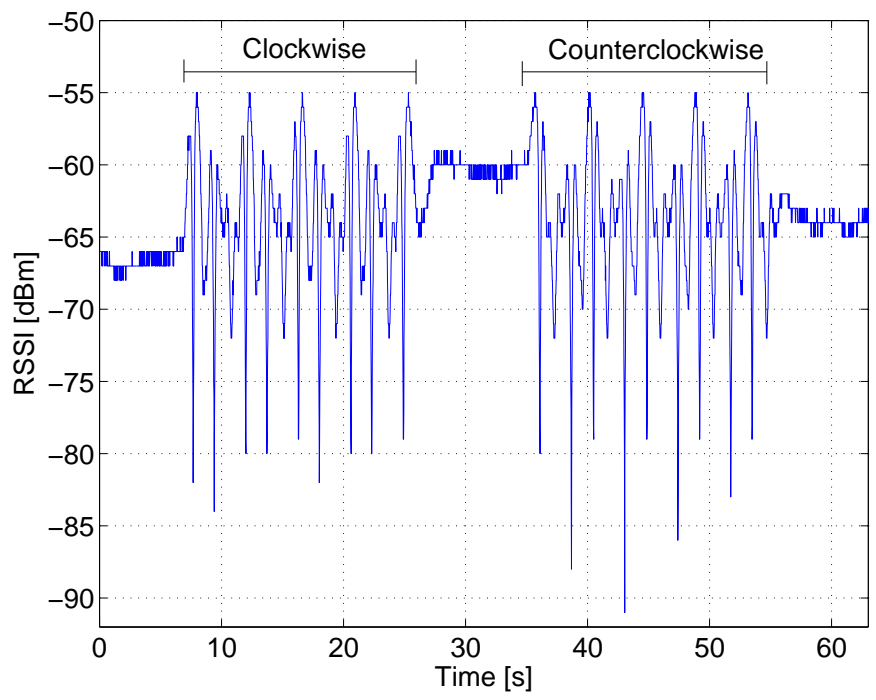

Fig. 13. Clockwise motion of a MICAz node on a turntable produces an RSSI pattern which is the time-reversal of the pattern produced by counterclockwise motion.

pointed out in [3]. However, in the absence of motion or other types of disturbances in the vicinity of the nodes (such as wind), RSSI levels are only affected by thermal noise and thus are expected to remain virtually constant. In the case of a motion event that does not modify the position of any node in the network, noticeable fluctuations affect the RSSI of a number of links; once the motion event terminates, RSSI levels return to their original state. Therefore, spatial memory properties are inherent to wireless links in networks with static nodes: given a particular topology, different layouts of the surrounding environment uniquely map to different sets of RSSI levels. Multipath fading is in fact a spatial phenomenon; we illustrate this point, often prone to misconceptions, with 


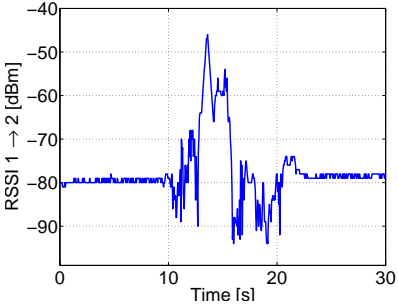

(a) RSSI for link $1 \rightarrow 2$.

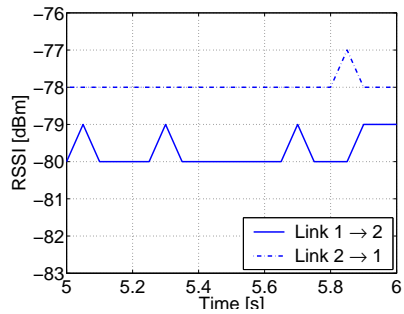

(c) RSSI before the swap.

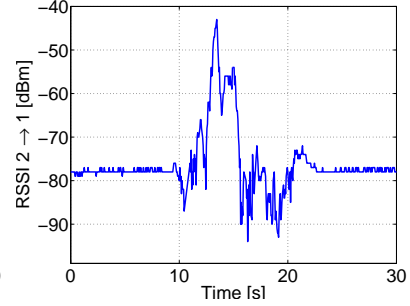

(b) RSSI for link $2 \rightarrow 1$.

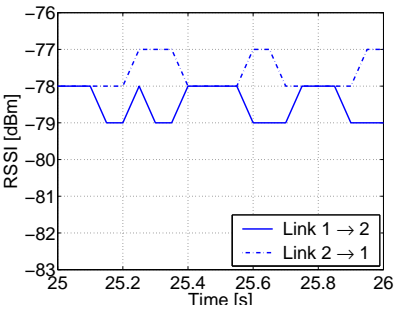

(d) RSSI after the swap.
Fig. 14. MICAz nodes 1 and 2 continuously transmit to each other and measure RSSI. Between time 10 and 20, the nodes are physically swapped, but the RSSI levels remain the same within $1 \mathrm{~dB}$, as can be seen by zooming in on the signal levels before (at time 5.5 in Fig. 14(c)) and after (at time 25.5 in Fig. 14(d)) the nodes are swapped.
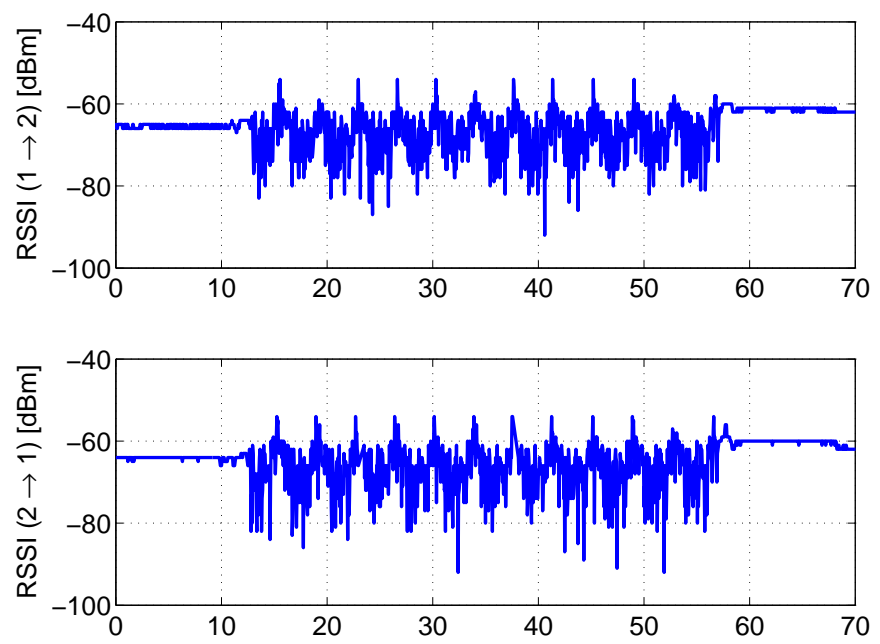

Fig. 15. Symmetry of wireless links during a turntable experiment.

a simple experiment. We place a MICAz transmitter on a motorized turntable, and measure RSSI at a static receiver. The turntable is first rotated in a clockwise and then in a counterclockwise direction. As shown in Fig. 13, the RSSI pattern corresponding to the latter is the time-reversal of the pattern corresponding to the former direction. The only discrepancies are due to sampling and quantization. The three static RSSI levels bracketing the rotation events are different as they correspond to three distinct positions of the transmitter on the turntable.

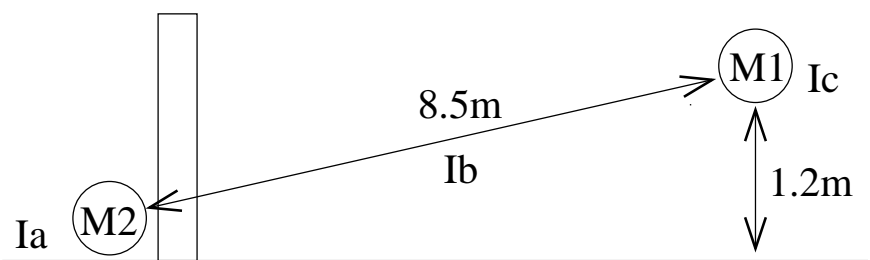

Fig. 16. Motes M1 and M2 are communicating in the presence of an interferer (which successively occupies positions Ia, Ib, and Ic).

\begin{tabular}{|l|l|l|l|l|}
\hline & No Interference & Ia & Ib & Ic \\
\hline Link $\mathbf{2} \rightarrow \mathbf{1}$ & $0.7 \%$ & $4.9 \%$ & $9.4 \%$ & $10 \%$ \\
\hline Link $\mathbf{1} \rightarrow \mathbf{2}$ & $0.4 \%$ & $10 \%$ & $9.7 \%$ & $1.3 \%$ \\
\hline
\end{tabular}

TABLE I

PACKET LOSS PROBABILITY WITHOUT AND WITH INTERFERENCE FOR THE EXPERIMENTAL LAYOUT SHOWN IN FIG. 16.

\section{B. Link symmetry}

In general, given two nodes $i$ and $j$, the channel between $i$ and $j$ is highly correlated with the channel between $j$ and $i$. In the experiment shown in Fig. 14, nodes 1 and 2 are physically swapped. The motion of the two nodes causes large variations in the signal strength patterns, but eventually the signal levels are within $1 \mathrm{~dB}$ of the starting levels. Fig. 15 shows the links between a static mote and a second mote on our turntable which is set to rotate at constant rate. The only discrepancies in the signal strength pertaining to the links are well within the accuracy of the measurements; such links are in fact virtually equivalent in terms of signal strength, and the probability of successful reception is approximately 1 for both.

Asymmetries may be introduced by radio irregularities [4] (for instance, the antennae are not perfectly isotropic) and, more importantly, by interference. Fig. 16 shows an experimental setup where a pair of motes (M1 and M2) send a packet to each other every $100 \mathrm{~ms}$ at a transmit power of $-10 \mathrm{dBm}$. A large metal obstacle and various objects block the LOS path in the proximity of M2. In the absence of interference, the packet loss probability for link $1 \rightarrow 2$ is virtually equivalent to the one for link $2 \rightarrow 1$, as shown in Table I. As an interferer transmitting every $15 \mathrm{~ms}$ at $0 \mathrm{dBm}$ is placed in position Ia, M2 suffers from the capture effect [2] and a strong asymmetry is introduced (link $1 \rightarrow 2$ is twice as lossy as link $2 \rightarrow$ 1). An even stronger asymmetry occurs when the interferer is located in position Ic: link $2 \rightarrow 1$ is lossier than link 1 $\rightarrow 2$ by one order of magnitude. Links are again symmetric (although obviously lossy) when the interferer is in position $\mathrm{Ib}$.

\section{Spatial memory experiments}

To illustrate the spatial memory concept, a network comprised of 4 MICAz nodes is deployed in a laboratory as 


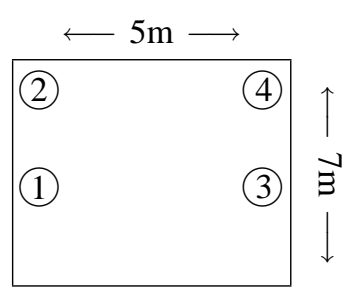

Fig. 17. Network layout for spatial memory experiments. The nodes are located approximately $50 \mathrm{~cm}$ from the nearest wall, and the distance between nodes 1 and 2 and nodes 3 and 4 is about $3.5 \mathrm{~m}$.
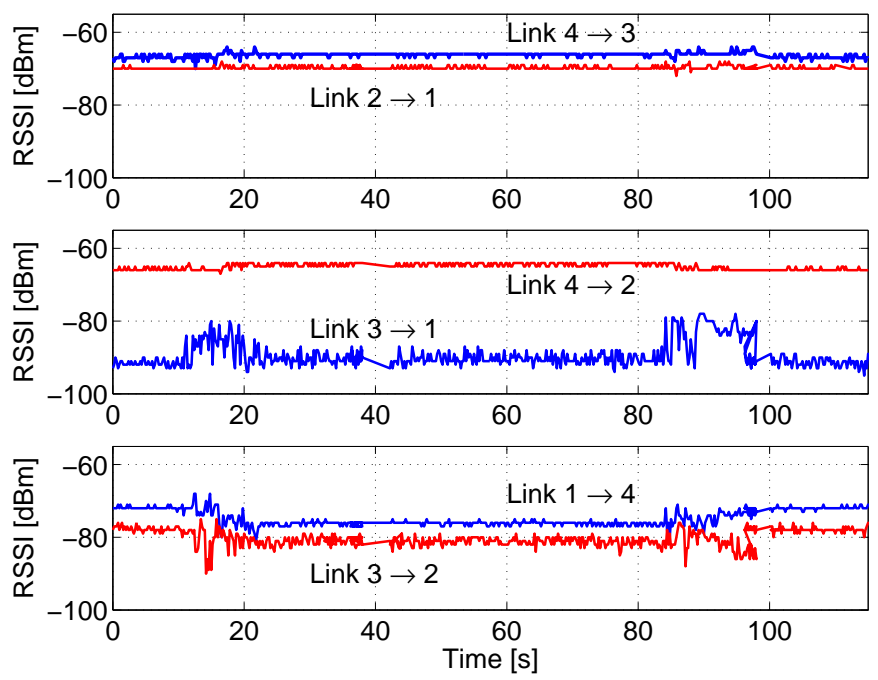

Fig. 18. An example of spatial memory: moving a chair. affects a number of links, which go back to their initial condition as the chair is returned to its original position.

shown in Fig. 17, and RSSI is measured on all wireless links. We employ a TDMA scheme at the MAC layer to avoid interference; by virtue of the aforementioned arguments, we can assume the wireless links in the network to be symmetric and only monitor one link for each pair of nodes. In Fig. 18, the layout of the room is modified by temporarily changing the position of a chair. Three links are affected $(3 \rightarrow 1,3 \rightarrow 2$, $1 \rightarrow 4$ ), but as the chair is returned to its original position, the RSSI levels also go back to their original values. In Fig. 19, the layout of the room is modified by the motion of two people. In this case, all links are affected (with the exception of $1 \rightarrow 2$ and $1 \rightarrow 3$ ), but as the people return to their original position, the RSSI levels corresponding to the initial layout are restored.

\section{CONCLUding REMARKS}

Multipath fading and shadowing have a strong impact on the wireless medium and certainly play a major role in the volatility of wireless links. These phenomena embed sensing capabilities into the RF signal by affecting its strength; the effects of shadowing and fading may therefore be constructively exploited in various ways. The most immediate sensorless sensing application is undoubtedly motion detection, as motion is the primary cause of signal fluctuations. Fading and shadowing are in fact spatial phenomena, and time variations are
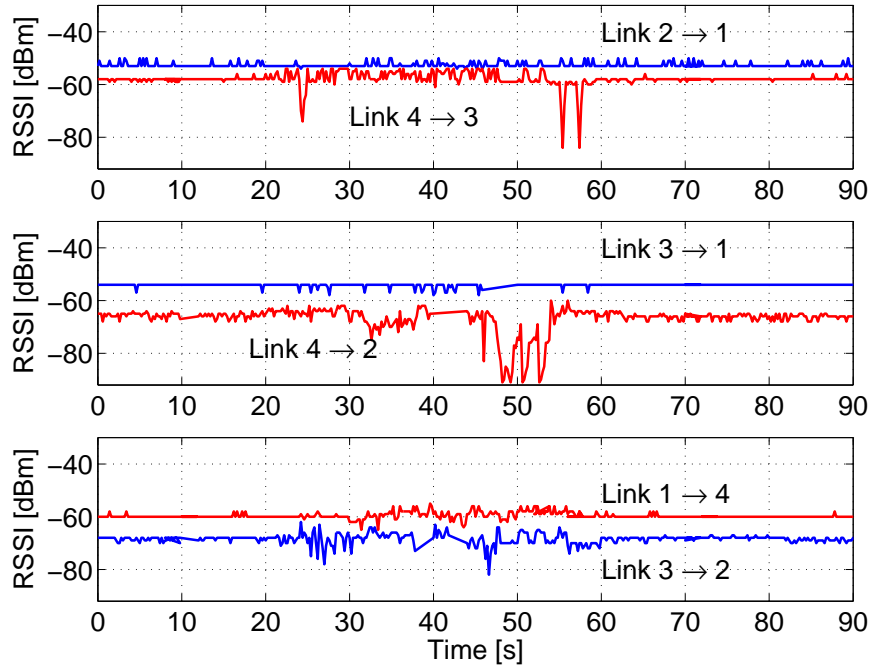

Fig. 19. Spatial memory: people moving in a room.

only introduced by dynamic changes in the network topology or the surrounding environment. Motion detection lays the foundation for a deeper degree of information harvesting targeted at velocity estimation. The spatial nature of fading further enriches the potential of a radio as a sensorless sensor: wireless links have intrinsic spatial memory properties, and can be used for the recognition of different configurations of the network and the surrounding environment. Although demonstrated here with MICA motes, these applications are not constrained to this platform; any platform operating in a rich scattering environment, including 802.11, will experience multipath fading and shadowing effects, and therefore will be capable of exploiting them in a similar manner.

In comparing our sensorless sensing method to more traditional approaches, we look at the tradeoff between cost and power. Sensorless sensing has an advantage in that it requires no additional hardware and therefore comes at no additional price; all that is required is a radio with embedded signal strength measurement capabilities. However, a comparison of power consumption has a less definitive result. The sensorless approach requires constant communication for proper operation while traditional sensors do not. However, sensorless sensing leverages on regular network operation. Finally, the power consumption in this approach depends on the transmission rate, which in turns depends on the application. Although the sensorless approach may not replace actual sensors, the addition of fading-induced information on the environment certainly has advantages in many applications. Last but not least, sensorless sensing has another important benefit: it provides a deeper understanding of wireless links and shows that it is possible to constructively exploit physical phenomena which are traditionally regarded as negative. 


\section{REFERENCES}

[1] J. Hill, R. Szewczyk, A. Woo, S. Hollar, D. Culler, and K. Pister. System Architecture Directions for Networked Sensors. In Proceedings of the 9th International Conference on Architectural Support for Programming Languages and Operating Systems (ASPLOS'O0), Cambridge, MA, Nov. 2000.

[2] K. Whitehouse, A. Woo, F. Jiang, J. Polastre, and D. Culler. Exploiting the capture effect for collision detection and recovery. In Proceedings of the 2nd IEEE Workshop on Embedded Networked Sensors (EmNetS-II), Sydney, Australia, May 2005.

[3] J. Zhao and R. Govindan. Understanding Packet Delivery Performance in Dense Wireless Sensor Networks. In Proceedings of the First International Conference on Embedded Networked Sensor Systems (SenSys03), Los Angeles, CA, Nov. 2003.

[4] G. Zhou, T. He, S. Krishnamurthy, and J. Stankovic. Impact of Radio Irregularity on Wireless Sensor Networks. In The Second International Conference on Mobile Systems, Applications, and Services, Boston, MA, June 2004. 